\title{
NOTAS SOBRE Xenolepidichthys dalgleishi GILCHRIST, 1922 (ACTINOPTERYGII: GRAMMICOLEPIDIDAE) AO LARGO DO ESTADO DA BAHIA, BRASIL, OCEANO ATLAANTICO OCIDENTAL
}

Notes on Xenolepidichthys dalgleishi Gilchrist, 1922 (Actinopterygii: Grammicolepididae) off Bahia State, Brazil, western Atlantic Ocean

Paulo Roberto Duarte Lopes ${ }^{1}$, Jailza Tavares de Oliveira-Silva², George Olavo

\begin{abstract}
RESUMO
São apresentados dados morfométricos e merísticos, e da dieta alimentar de Xenolepidichthys dalgleishi Gilchrist, 1922 (Actinopterygii: Grammicolepididae), com base em nove indivíduos capturados ao largo do Estado da Bahia, Brasil, Oceano Atlântico ocidental. O comprimento total variou de 125,6 mm a 160,0 mm. Pequenas variações foram observadas com relação aos caracteres merísticos e proporções corporais. A deita alimentar de X. dalgleishi consiste principalmente de peixes e crustáceos.
\end{abstract}

Palavras-chaves: Xenolepidichthys dalgleishi, caracteres merísticos, caracteres morfométricos, dieta alimentar, Estado da Bahia.

\begin{abstract}
Information on meristic and morphometric characteristics, and feeding diet of Xenolepidichthys dalgleishi Gilchrist, 1922 (Actinopterygii: Grammicolepididae), based on the catch of nine specimens off Bahia State, Brazil, western Atlantic Ocean), is presented. Total length ranged from $125.6 \mathrm{~mm}$ to $160.0 \mathrm{~mm}$. Slight variations were observed with respect to meristic characters and body proportions. The feeding diet of $\mathrm{X}$. dalgleishi consists mainly of fish and crustaceans.
\end{abstract}

Keywords: Xenolepidichthys dalgleishi, meristic characters, morphometric characters, feeding diet, Bahia State.

\footnotetext{
${ }^{1}$ Professor Assistente, Universidade Estadual de Feira de Santana, Departamento de Ciências Biológicas, BR-116 km 03, Feira de Santana, BA 44036-900. E-mail: andarilho40@gmail.com

2 Bióloga, Universidade Estadual de Feira de Santana, Departamento de Ciências Biológicas, BR-116 km 03, Feira de Santana, BA 44036-900. E-mail: jtosilva@yahoo.com.br

${ }^{3}$ Professor Assistente, Universidade Estadual de Feira de Santana, Departamento de Ciências Biológicas, BR-116 km 03, Feira de Santana, BA 44036-900. E-mail: georgeolavo@uol.com.br
} 


\section{INTRODUÇÃO}

Xenolepidichthys dalgleishi Gilchrist, 1922 (Figura 1), pertencente à família Grammicolepididae (ordem Zeiformes) e única espécie do gênero, atinge pelo menos $26,0 \mathrm{~cm}$ de comprimento. Habita sobre o fundo onde é capturada com rede-de-arrasto entre 90 e $900 \mathrm{~m}$ de profundidade, sendo citada para o Atlântico e Indo-Pacífico ocidental. No Brasil, é conhecida nas regiões Sudeste e Sul (Menezes, Figueiredo, 1980; Shimizu, 1983; Karrer \& Heemstra, 1986; Cervigón, 1991; McEachran \& Fechhelm, 1998; Heemstra, 2002; Menezes, 2003; Bernardes et al., 2005; Nelson, 2006).

\section{MATERIAL E MÉTODOS}

Foram examinados 9 exemplares de X. dalgleishi que se encontram depositados na coleção da Divisão de Peixes do Museu de Zoologia da Universidade Estadual de Feira de Santana (Bahia), conservados em álcool $70 \%$, registrados sob o número LIUEFS 15847, e que foram coletados com rede de arrasto de fundo pelo "R.V. Thalassa" durante a Operação Bahia-2, do Programa REVIZEE, na posição de $011^{\circ} 37^{\prime} \mathrm{S}-037^{\circ} 12^{\prime} \mathrm{W}$, em 466 metros de profundidade.

Cada exemplar foi dissecado para identificação do sexo e de um possível estágio de maturação através do exame das gônadas e retirada do tubo digestivo para exame do seu conteúdo sob microscópio estereoscópico.

Dados morfométricos foram obtidos no lado direito do corpo com paquímetro com precisão de $0,05 \mathrm{~mm}$ exceto, em alguns casos, comprimentos total e padrão quando foi utilizada régua com precisão de 1,0 mm e ictiômetro (Figura 1).

\section{RESULTADOS}

Os dados sobre os caracteres meríticios e morfométricos estão apresentados na Tabela I, dos quais foram estimadas as seguintes relações biométricas: $\mathrm{AC} / \mathrm{CP}=0,77-0,88 ; \mathrm{CC} / \mathrm{CP}=0,28-0,36$; $\mathrm{D}) / \mathrm{CC}=0,44-0,52$. Foram identificados 4 fêmeas $(44,4 \%), 4$ machos $(44,4 \%)$ e 1 indivíduo $(11,1 \%)$ cujo sexo não pode ser determinado com base no exame direto das gônadas.

Foram encontrados 3 estômagos vazios $(33,3 \%)$. O volume ingerido de alimento variou entre 0,1 e $0,8 \mathrm{ml}$.

Foram identificadas duas categorias alimentares, com predominância da presença de matéria orgânica digerida. Dentre os itens alimentares, destacam-se os peixes Actinopterygii Teleostei, com 33,3\% (83\% de frequência numérica), e os crustáceos da ordem Decapoda (representados por um par de olhos pedunculados), com 11,1\% (16,7\% de frequência numérica).

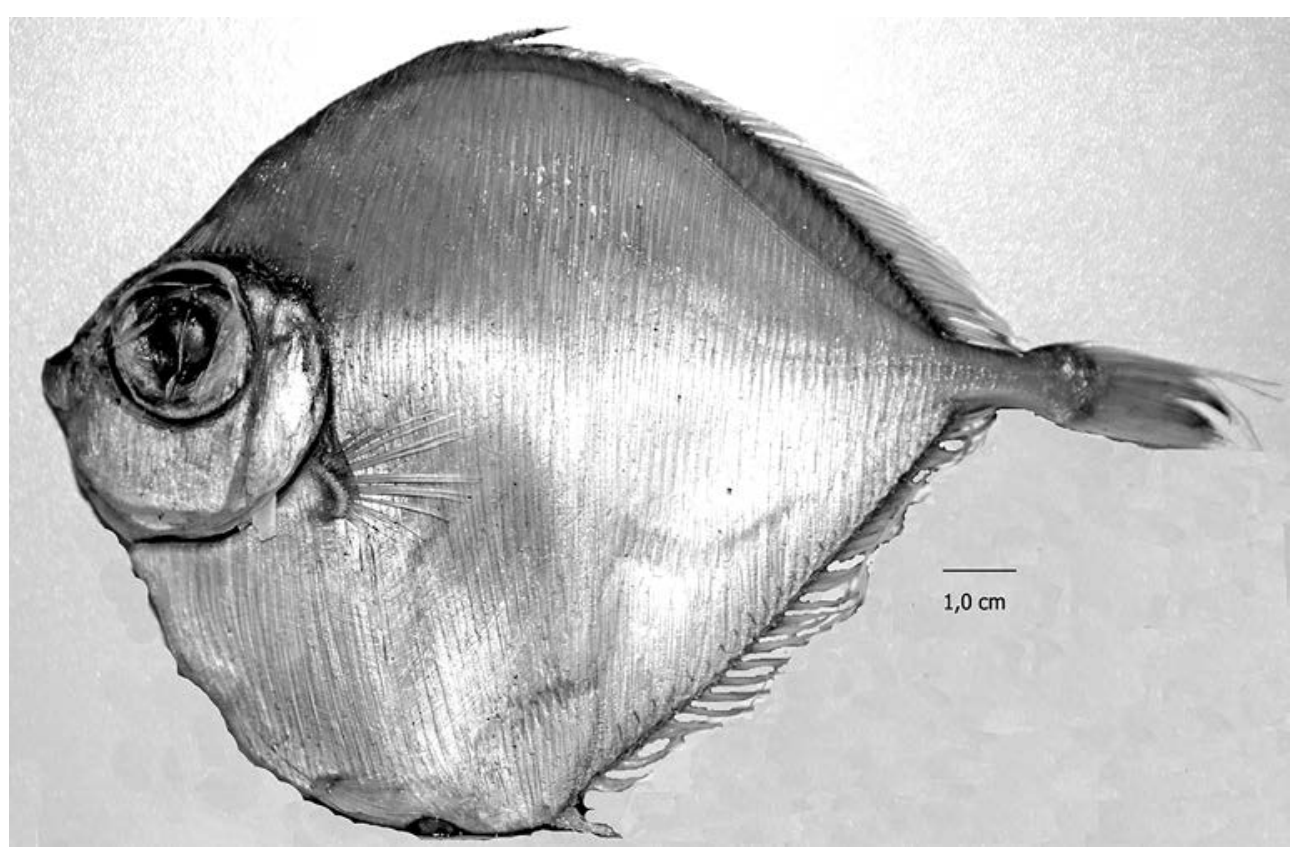

Figura 1 - Xenolepidichthys dalgleishi Gilchrist, 1922 (LIUEFS 15847). 
Tabela I - Dados sobre os caracteres merísticos e morfométricas coletados para nove indivíduos de Xenolepidichthys dalgleishi Gilchrist, 1922, ao largo do Estado da Bahia, Brasil.

\begin{tabular}{c|c|c|c|c|c|c|c|c|c|c|c}
\hline \multicolumn{1}{c}{ Caracteres merísticos } & \multicolumn{5}{c}{ Caracteres morfométricos } \\
\hline ED & RD & EA & RA & RPT & EP $^{1}$ & RPL $^{1}$ & CT & CP & AC & CC & DO \\
\hline $3^{*}$ & 30 & 2 & 29 & 14 & 1 & 5 & 160,0 & 118,9 & 99,1 & 43,4 & 20,3 \\
3 & 29 & 2 & 27 & 14 & 1 & 6 & 150,0 & 115,1 & 89,1 & 38,8 & 17,0 \\
4 & 26 & 2 & 28 & 14 & 1 & 5 & 127,0 & 94,5 & 82,8 & 62,6 & 16,5 \\
4 & 28 & 2 & 28 & 14 & 1 & $6,5^{2}$ & 145,0 & 111,5 & 91,0 & 37,3 & 17,4 \\
5 & 29 & 2 & 28 & 14 & 1 & 6 & 153,0 & 121,1 & 99,1 & 40,3 & 18,6 \\
$3^{*}$ & 28 & 2 & 28 & 14 & 1 & 6 & 125,6 & 93,8 & 82,5 & 31,0 & 15,0 \\
4 & 30 & 2 & 28 & 14 & 1 & 6 & 132,2 & 104,5 & 80,5 & 32,9 & 15,3 \\
4 & 28 & 2 & 28 & 12,14 & 1 & 6 & 130,6 & 107,9 & 88,3 & 33,5 & 17,3 \\
$3^{*}$ & 28 & 2 & 28 & 14 & 1 & 6 & 137,6 & 106,9 & 87,1 & 30,4 & 15,4 \\
\hline
\end{tabular}

Legenda:: $\mathrm{ED}=$ espinho dorsal; $\mathrm{RD}=$ raio dorsal; $\mathrm{EA}=$ espinho anal; $\mathrm{RA}=$ raio anal; $\mathrm{RPT}=$ raio peitoral; $\mathrm{EP}=$ espinho pélvico; $\mathrm{RPL}=$ raio pélvico; $\mathrm{CT}=$ comprimento total; $\mathrm{CP}=$ comprimento padrão; $\mathrm{AC}=$ altura do corpo; $\mathrm{CC}=$ comprimento da cabeça; $\mathrm{DO}=$ diâmetro orbital.

Observações: * = danificado; 1 = número igual nos lados direito e esquerdo; 2 = lados direito e esquerdo.

\section{DISCUSSÃO}

Segundo Karrer \& Heemstra (1986), Cervigón et al. (1992) e Heemstra (2002), a família Grammicolepididae compreende dois gêneros (com uma espécie cadae), demersais e cosmopolitas (mas não em mares polares). Não são comuns e nem de interesse comercial, ocorrendo numa variedade de ambientes entre 100 e $800 \mathrm{~m}$ de profundidade. Geralmente são capturados próximos ao fundo com redes-de-arrasto, mas nada tem sido publicado sobre sua biologia.

A outra espécie da família, conforme reconhecida por Nelson (2006), é Grammicolepis brachiusculus Poey, 1873, registrada no Brasil ao largo do estado da Bahia (Costa et al., 2007; Carvalho-Filho et al., 2012).

$X$. dalgleishi esteve entre as espécies mais abundantes entre 251 e $522 \mathrm{~m}$ de profundidade, no talude superior ao norte do Banco dos Abrolhos (entre $11^{\circ} \mathrm{S}$ e $15^{\circ} \mathrm{S}$ ), entre maio e julho de 2000 (Costa et al., 2007).

A altura do corpo está contida de 0,8 a 1,2 vezes e o comprimento da cabeça de 2,9 a 3,7 vezes no comprimento padrão, enquanto o olho está contido de 2,0 a 2,5 vezes no comprimento da cabeça em indivíduos de comprimento padrão entre 5,0 e 12,0 cm (Karrer \& Heemstra, 1986; Heemstra, 2002).

O número de espinhos e raios nas diferentes nadadeiras coincidiu os valores obtidos por esses autores, com divergência observada apenas no número de espinhos da nadadeira dorsal. Com relação às proporções corporais, pequenas variações foram observadas nas relações altura do corpo/comprimento padrão, comprimento da cabeça/comprimento padrão e diâmetro orbital/comprimento da cabeça.
A pequena amostra aqui examinada não permite conclusões sobre a biologia de $X$. dalgleishi, mas contribui para ampliar os conhecimentos sobre esta espécie ao longo de sua área de ocorrência no Oceano Atlântico ocidental.

Agradecimentos - À coordenação do Programa REVIZEE/SCORE Central e ao Prof. Dr. Gustavo W. Nunan (UFRJ, MNRJ) (†) pela liberação do material aqui analisado para incorporação na coleção de peixes do Museu de Zoologia da UEFS. À Bahia Pesca S.A. e à UEFS pelo apoio logístico para o embarque do primeiro e do terceiro autor no "R.V.Thalassa", e à sua tripulação pelo auxílio a bordo.

\section{REFERÊNCIAS BIBLIOGRÁFICAS}

Bernardes, R.A.; Figueiredo, J.L.; Rodrigues, A.R.; Fischer, L.G,; Vooren, C.M., Haimovici, M. \& RossiWongtschowski, C.L.B. Peixes da Zona Econômica Exclusiva da região Sudeste-Sul do Brasil. Editora da Universidade de São Paulo, 295 p., São Paulo, 2005.

Carvalho-Filho, A.; Marcovaldi, G.; Ribeiro, F.A.; Paiva, M.I.G. \& Sampaio, C.L.S. New records of Grammicolepis brachiusculus Poey, 1873 (Zeiformes: Grammicolepididae) in Brazilian waters, with a key to western Atlantic species of Grammicolepididae. Check List, v.8, n.4, p.626-629, 2012.

Cervigón, F. Los peces marinos de Venezuela. Vol .I. Fundación Científica Los Roques, $2^{\text {a }}$ edición, 425 p., Caracas, 1991.

Cervigón, F.; Cipriani, R.; Fischer, W.; Garibaldi, L.; Hendrickx, M.; Lemus, A.J.; Márquez, R.; Poutiers, 
J.M.; Robaina, G. \& Rodriguez, B. Guia de campo de las espécies comerciales marinas y de águas salobres de la costa septentrional de Sur America. FAO, 513 p., Roma, 1992.

Costa, P.A.S.; Braga, A.C.; Melo, M.R.S.; Nunan, G.W.A.; Martins, A.S. \& Olavo, G. Assembléia de teleósteos demersais no talude da costa central brasileira, p. 87-107, in Costa, P.A.S., Olavo, G. \& Martins, A.S. (eds.), Biodiversidade da fauna marinha profunda na costa central brasileira. Museu Nacional (Série Livros no. 24), 183 p., Rio de Janeiro, 2007.

Heemstra, P.C. Grammicolepidae, p. 1214-1216, in Carpenter, K. (ed.), The living marine resources of the Western Central Atlantic. Volume 2: Bony fishes - Part 1 (Acipenseridae to Grammatidae). FAO Species Identification Guide for Fishery Purposes and American Society of Ichthyologists and Herpetologists Special Publication, n. 5, Rome, 2002.

Karrer, C. \& Heemstra, P.C. Family no. 140: Grammicolepididae, p. 440-441, in Smith, M.M. \&
Heemstra, P.C. (eds.), Smiths' sea fishes. SpringerVerlag, 1047 p., Berlin, 1986.

McEachran, J.D. \& Fechhelm, J.D. Fishes of the Gulf of Mexico. Volume 1: Myxiniformes to Gasterosteiformes. University of Texas, 1112 p., Austin, 1998.

Menezes, N.A. Família Grammicolepididae, p. 70, in Menezes, N.A., Buckup, P.A., Figueiredo, J.L. \& Moura, R.L. (eds.), Catálogo das espécies de peixes marinhos do Brasil. Museu de Zoologia da Universidade de São Paulo, 159 p., São Paulo, 2003.

Menezes, N.A. \& Figueiredo, J.L. Manual de peixes marinhos do sudeste do Brasil. III. Teleostei (2). Museu de Zoologia da Universidade de São Paulo, 90 p., São Paulo, 1980.

Nelson, J.S. Fishes of the world. John Wiley \& Sons, $4^{\text {th }}$ edition, 601 p., New Jersey, 2006.

Shimizu, T. Grammicolepididae, p. 291, in Uyeno, T., Matsuura, K. \& Fujii, E. (eds.), Fishes trawled off Suriname and French Guiana. Japan Marine Fishery Resource Research Center, 519 p., Tokyo, 1983. 